\title{
Aumento en la positividad del biomarcador galactomanano durante la pandemia de COVID-19 en la Quinta Región: Valparaíso
}

\author{
Increase in the positivity of the galactomannan biomarker during the COVID-19 \\ pandemic in the Fifth Region: Valparaíso
}

Rodrigo Cruz Choappa',2, Peggy Vieille², Yerko Leiva², Melissa Noguera² y Eduardo López

'Infectología y Laboratorio de Micología. Universidad de Valparaíso. Valparaíso, Chile.

${ }^{2}$ Laboratorio de Micología. Universidad de Valparaíso. Valparaíso, Chile.

Los autores no tienen conflictos de interés.

Financiamiento: Universidad de Valparaíso.

Recibido: 16 de octubre de 2020 / Aceptado: 18 de marzo de 2021

\section{Resumen}

Introducción: La actual pandemia provocada por SARS-CoV-2 ha provocado una alta carga en la salud pública y privada. Se han descrito casos y series de aspergilosis invasora asociada a pacientes con COVID-19 en ventilación mecánica. Objetivo: Describir el aumento en la positividad del biomarcador galactomanano (GM) durante la pandemia de COVID-19 en la Quinta Región: Valparaíso. Materiales y Método: Estudio descriptivo, retrospectivo. Se revisó la cantidad y los resultados de GM, tanto de lavado bronco-alveolar (LBA) como en suero y los cultivos de LBA enviados al laboratorio de Micología de la Universidad de Valparaíso, desde enero y hasta septiembre del año 2020; luego se compararon con los exámenes recibidos en el mismo período del año 2019. Resultados: Se observó un aumento significativo de los GM realizados en LBA, concentrándose principalmente entre los meses de julio y septiembre. El $29 \%$ de las muestras del año 2020 tenía el antecedente de ser de pacientes con COVID-19. Del total de muestras positivas durante el año de la pandemia, 5/12 fueron en pacientes con COVID-19. Conclusiones: Hubo un aumento significativo de los GM realizados en LBA durante la pandemia, concentrándose principalmente entre los meses de julio-septiembre.

Palabras clave: aspergilosis invasora; diagnóstico; SARS-CoV-2.

\section{Abstract}

Background: The current pandemic due to SARS-CoV-2 has caused a high burden on health. Cases and series of invasive aspergillosis associated with COVID-19 patients (CAPA) on mechanical ventilation have been described. Aim: To describe the increase in the positivity of the galactomannan (GM) biomarker during the COVID-19 pandemic in the Fifth Region: Valparaíso. Method: Retrospective descriptive study. The GM results in both bronchoalveolar lavage (BAL) and serum and the BAL cultures that were sent to the Mycology Laboratory of the University of Valparaíso from January to September 2020 were reviewed; then they were compared with the examinations of the same period of 2019. Results: There was a significant increase in GMs carried out in LBA during the pandemic, concentrating mainly between the months of July-September. Conclusions: There was a significant increase in GM carried out in LBA during the pandemic, concentrating mainly between the months of July-September.

Keywords: invasive aspergillosis; diagnosis; SARS-CoV-2. 


\section{Introducción}

\section{L} a aspergilosis invasora $(\mathrm{AI})$ es una infección grave que afecta con mayor frecuencia a pacientes con algún grado de inmunocompromiso celular, como neutropenia prolongada, trasplante de progenitores hematopoyéticos o de órgano sólido, uso de corticoesteroides, SIDA y enfermedad injerto contra hospedero ${ }^{1}$. Sin embargo, también se asocia a pacientes con influenza, EPOC o cirróticos que se encuentran en ventilación mecánica ${ }^{1,2}$. La actual pandemia provocada por SARS-CoV-2 ha provocado una alta carga de morbilidad, mortalidad y de costos al sistema sanitario en todo el mundo ${ }^{3,4}$. Recientemente, Bartoletti M. y cols., han descrito una serie de casos de AI asociada a pacientes con COVID-19 (sigla en inglés CAPA) en ventilación mecánica, en cuya propuesta diagnóstica se incluye la presencia de infiltrados pulmonares más uno de los siguientes criterios: índice de galactomanano (GM) en suero $>0,5$, GM en lavado bronco-alveolar $(\mathrm{LBA})>1,0$, un cultivo con desarrollo de Aspergillus spp en LBA, o la presencia de cavitación del parénquima no atribuido a otra causa ${ }^{5}$. CAPA ocurre generalmente después del cuarto día de la intubación y desde los catorce días de iniciado los síntomas de COVID-19. El factor asociado significativamente en todos los casos es el uso de corticosteroides ${ }^{5}$. La mortalidad a los 30 días es significativamente mayor en aquellos con este diagnóstico que en aquellos sin él (44 versus 19\%) y aumenta 1,41 veces por cada punto en el índice GM inicial ${ }^{5}$. Hasta el momento no se han publicado series de casos de CAPA en Chile o en Latinoamérica. El objetivo del presente trabajo es describir el aumento en la positividad del biomarcador GM durante la pandemia de COVID-19 en la Quinta Región: Valparaíso.

\section{Materiales y Método}

Estudio descriptivo, retrospectivo. Se revisó la cantidad y los resultados de GM, tanto de LBA como en suero y los cultivos de LBA enviados desde cuatro hospitales de la región (Dr. Gustavo Fricke de Viña del Mar, Carlos van Buren de Valparaíso, San Martín de Quillota e Instituto de Seguridad del Trabajo-IST Viña del Mar) al laboratorio de Micología de la Universidad de Valparaíso desde enero hasta septiembre del año 2020; luego se compararon con los exámenes del mismo período del año 2019. Además, se obtuvieron algunos datos demográficos y antecedentes de COVID-19.

El índice de GM se midió con un ensayo inmunoenzimático tipo sándwich (Platelia ${ }^{\mathrm{TM}}$ Aspergillus Ag; BioRad), según las indicaciones del fabricante. Las muestras de LBA se cultivaron en agar Sabouraud con cloranfenicol a $37^{\circ} \mathrm{C}$ durante 10 días, revisándolos diariamente para determinar si había desarrollo de hongos. La identifica- ción de las especies se realizó mediante pruebas morfofisiológicas estandarizadas para cada género y especie.

\section{Análisis de datos}

Los datos fueron tabulados en planilla Excel y se expresaron en gráficos de barras comparando los números entre los meses de los años estudiados. Los resultados se expresaron en números absolutos y en porcentajes. Para determinar la significancia estadística entre ambos años estudiados se utilizó la prueba de proporciones de dos muestras en STATA 15.0 (StataCorp).

\section{Resultados}

Las muestras enviadas correspondieron a pacientes de 45 y 46 años de edad promedio en los años 2019 y 2020 , respectivamente. El 64 y $55 \%$ de las muestras fueron de pacientes de sexo masculino en los mismos años estudiados. El 29\% de los pacientes a quienes se les solicitó GM en el año de la pandemia tenían el antecedente de estar cursando con COVID-19.

Durante el año de la pandemia se observó un aumento de las solicitudes de detección de GM, principalmente de LBA (Figura 1), siendo estadísticamente significativo (p: 0,015). En el caso de las muestras de suero, esta diferencia no fue significativa (p: 0,59).

El mayor número de solicitudes de GM en LBA se concentró entre julio y septiembre (Figura 2).

Del total de muestras positivas durante el año de la pandemia, 5/12 fueron en muestras de pacientes con el antecedente de COVID-19 que se encontraban en ventilación mecánica (Tabla 1).

Del total de las muestras de LBA sembradas, hubo desarrollo principalmente de Candida spp (Tabla 2).

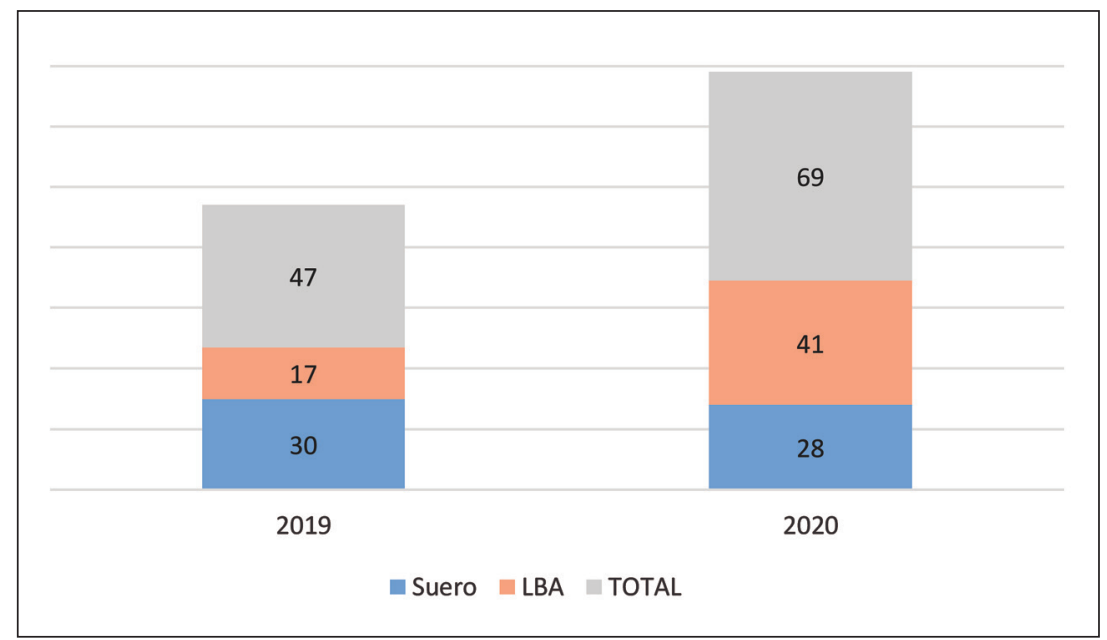

Figura 1. $\mathrm{N}^{\circ}$ de test realizados (período enero-septiembre 2019-2020). Se observa un aumento de las muestras para GM en LBA durante la pandemia. 


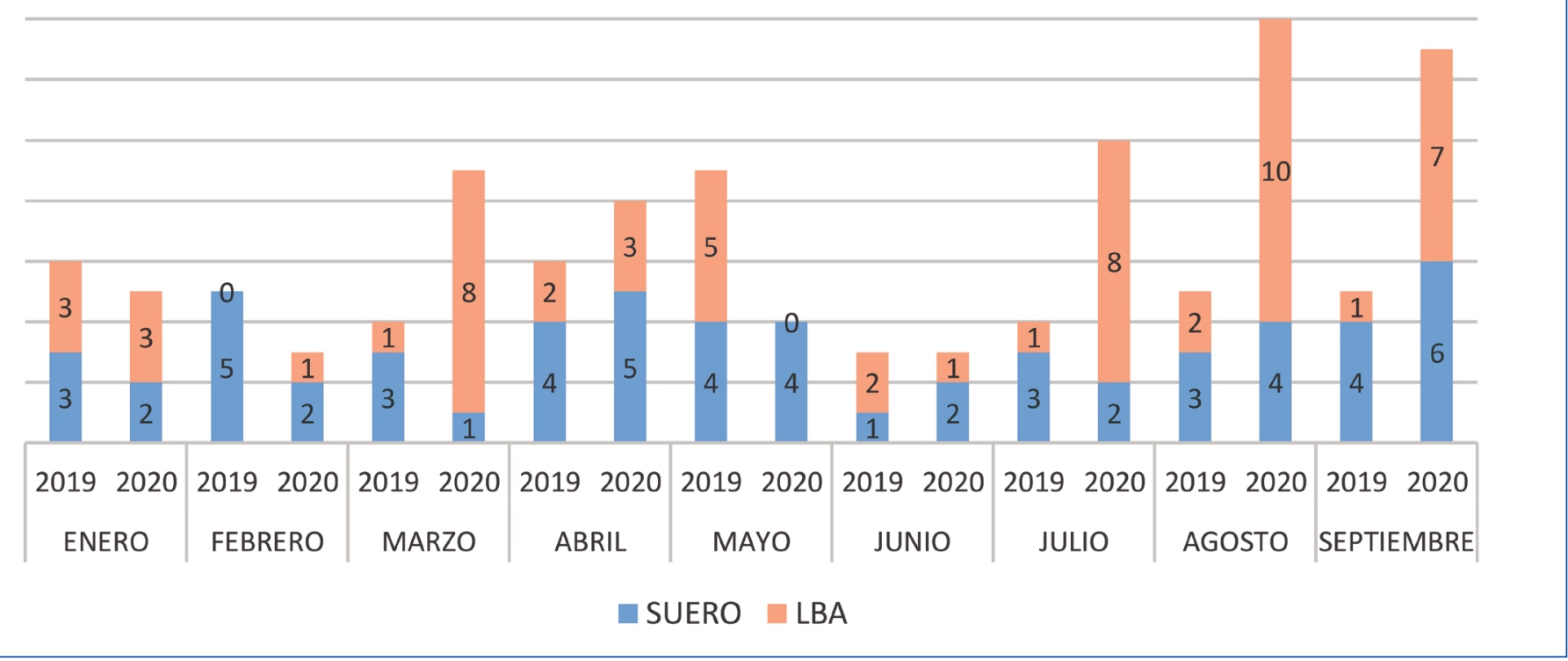

Figura 2. Comparación mensual por tipo de muestra (Período enero-septiembre 2019/2020). Se observa como aumentó la solicitud de GM desde julio.

Tabla 1. Muestras positivas según pacientes. Período año 2020

\begin{tabular}{cccc} 
Tipo de muestra & COVID-19 & No COVID-19 & Total \\
Suero & 0 & 2 & 2 \\
LBA & 5 & 5 & 10 \\
\hline
\end{tabular}

\begin{tabular}{lccc|}
\hline Tabla 2. Especies aisladas desde LBA & & \\
\hline & COVID-19 & No COVID -19 & Total \\
Negativo & 6 & 20 & 26 \\
\hline Candida spp & 9 & 5 & 14 \\
\hline Aspergillus fumigatus & 1 & 0 & 1
\end{tabular}

\section{Discusión}

El 30 de enero del año 2020, la Organización Mundial de la Salud (OMS) anunció un nuevo brote de coronavirus (SARS-CoV-2) que rápidamente se diseminó en el mundo, declarándose el 11 de marzo como pandemia ${ }^{6}$.

El SARS- CoV-2 es el agente responsable de causar la enfermedad COVID-19 y tuvo su origen en la ciudad de Wuhan, China ${ }^{7}$.

Hasta la fecha, este virus ha provocado más de 35 millones de infectados y más de 1 millón muertos en todo el mundo ${ }^{6,8}$. Chile ha sido fuertemente afectado, a la fecha de este envío, con cerca de 500 mil infectados y más de 17 mil muertos por causa directa o indirecta de esta infección ${ }^{8,9}$.

La AI clásicamente se ha asociado a pacientes con patología onco-hematológica; sin embargo, también se describen casos en pacientes con otras patologías o factores de riesgo ${ }^{10,11}$. La definición de CAPA incluye un valor de GM en suero $>0,5, \mathrm{GM}$ en LBA $>1,0$ o cultivo con desarrollo de Aspergillus spp en LBA o alguna cavitación no atribuido a otra causa. En nuestro trabajo, los valores de $G M \geq 0,5$ en suero $\mathrm{y} \geq 0,8$ en LBA se consideraron como positivos, ambos incluidos dentro de la actualización de las definiciones del consenso de enfermedad fúngica invasora según EORTC / $\mathrm{MSG}^{12}$. GM en suero y en LBA se recomienda como un marcador con una alta sensibilidad y especificidad para el diagnóstico de AI probable en pacientes adultos y pediátricos, especialmente en aquellos con neoplasia maligna hematológica y trasplante de progenitores hematopoyéticos; sin embargo, la utilidad en otras patologías como COVID-19, aún no está bien definida ${ }^{1,10,11}$. En nuestro trabajo hubo un aumento de las solicitudes de GM durante el 2020 debido a pacientes con antecedente de COVID-19 en ventilación mecánica, lo que puede indicar que se ha realizado una búsqueda activa de CAPA en algunos hospitales de la región.

Se han descrito casos de CAPA en distintos luga- 
nuestra población por un tiempo aún no bien conocido, por lo que debemos tener presente que en aquellos pacientes graves que se encuentran en ventilación mecánica; una de las complicaciones posibles es CAPA, para lo cual debemos sospecharla y diagnosticarla precozmente para evitar un desenlace fatal.

\section{Conclusiones}

Las muestras de nuestro estudio fueron principalmente de pacientes hombres jóvenes. Durante el año de la pandemia hubo un aumento del número de GM solicitados y de los resultados positivos, principalmente en LBA, concentrándose entre los meses de julio-septiembre. Del total de muestras positivas durante el año 2020, más de $40 \%$ fueron en muestras de pacientes con COVID-19 que se encontraban en ventilación mecánica. En la mayoría de los cultivos de LBA no hubo desarrollo de hongos filamentosos.

\section{Referencias bibliográficas}

1.- Patterson T, Thompson G, Denning D, Fishman J, Hadley S, Herbrecht R, et al. Practice Guidelines for the Diagnosis and Management of Aspergillosis: 2016 Update by the Infectious Diseases Society of America. Clin Infect Dis. 2016; 63 (4): e1-e60. https://doi.org/10.1093/ cid/ciw326.

2.- Doligalski C T, Benedict K, Cleveland A A, Park B, Derado G, Pappas P, et al. Epidemiology of invasive mold infections in lung transplant recipients. Am J Transplant. 2014; (14): 1328-33. https://doi.org/10.1111/ ajt.12691.

3.- Huang C, Wang Y, Li X, Ren L, Zhao J, Hu $\mathrm{Y}$, et al. Clinical features of patients infected with 2019 novel coronavirus in Wuhan, China. Lancet 2020; 395 (10223): 497-506. https://doi. org/10.1016/S0140-6736(20)30183-5.

4.- Grasselli G, Pesenti A, Cecconi M. Critical care utilization for the COVID-19 outbreak in Lombardy, Italy: early experience and forecast during an emergency response. JAMA 2020; 323 (16): 1545-6. doi:10.1001/jama.2020.4031.

5.- Bartoletti M, Pascale R, Cricca M, Rinaldi M, Maccaro A, Bussini L, et al. Epidemiology of invasive pulmonary aspergillosis among COVID-19 intubated patients: a prospective study. Clin Infect Dis. 2020; 1-9. https://doi org/10.1093/cid/ciaa1065.

6.- WHO Director-General's opening remarks at the media briefing on COVID-19 - 11 March 2020. Available from: https://www.who.int/ $\mathrm{dg} /$ speeches/detail/who-director-general-sopening-remarks-at-the-media-briefing-oncovid-19---11-march-2020.

7.- Abarca B, Vargas J, García J. Caracterización patogénica, clínica y diagnóstica de la pandemia por SARS-CoV-2. Rev Chilena Infectol. 2020; 37 (3): 265-75. http://dx.doi. org/10.4067/s0716-10182020000300265.

8.- Johns Hopkins University and Medicine.https:// coronavirus.jhu.edu/map.html. (Consultado el 6 de octubre de 2020).

9.- Informe epidemiológico $N^{0} 56$. Enfermedad SARS-CoV-2 (COVID-19). Chile 2-102020. https://www.minsal.cl/wp-content/ uploads/2020/10/Informe-Epidemiologico-56. pdf.

10.- Baddley J W, Stephens J M, Ji X, Gao X, Schlamm H T, Tarallo M. Aspergillosis in intensive care unit (ICU) patients: epidemiology and economic outcomes. BMC Infect Dis. 2013; 13: 29. doi: 10.1186 / 14712334-13-29.

11.- Blot S I, Taccone F S, Van den Abeele A M, Bulpa P, Meersseman W, Brusselaers W. A clinical algorithm to diagnose invasive pulmonary aspergillosis in critically ill patients. Am J Respir Crit Care Med. 2012; 186: 56-64. https://doi.org/10.1164/ rccm.201111-1978oc.

12.- Donnelly J P, Chen S C, Kauffman C
A, Steinbach W J, Baddley J W, Verweij P E, et al. Revision and Update of the Consensus Definitions of Invasive Fungal Disease From the European Organization for Research and Treatment of Cancer and the Mycoses Study Group Education and Research Consortium. Clin Infect Dis. 2020; 71 (6): 1367-76. doi: 10.1093 / cid / ciz1008.

13.- Van Arkel A L E, Rijpstra T A, Belderbos H N A, Van Wijngaarden P, Verweij P E, Bentvelsen R G. COVID-19-associated pulmonary aspergillosis. Am J Respir Crit Care Med. 2020; 202 (1): 132-5. doi:10.1164/rccm.2020041038LE.

14.- Helleberg M, Steensen M, Arendrup M C. Invasive aspergillosis in patients with severe COVID-19 pneumonia. Clin Microbiol Infect. 2021; 27 (1): 147-148. doi: 10.1016/j. cmi.2020.07.047

15.- Santana M, Guilherme P, Araujo A M, Baía-da-Silva D C, da Silva Borba M G, Almeida Val F, et al. Confirmed invasive pulmonary aspergillosis and COVID-19: the value of postmortem findings to support antemortem management. Rev Soc Bras Med Trop. 2020; 53: e20200401. https://doi. org/10.1590/0037-8682-0401-2020.

16.- Cruz R. Guía para el diagnóstico de laboratorio de enfermedad fúngica invasora por hongos filamentosos. Rev Chilena Infectol. 2014; 31 (2): 173-9. http://dx.doi.org/10.4067/S071610182014000200008 . 